\title{
GEOMETRICAL PROPERTIES OF EQUIPOTENTIAL SURFACES
}

\section{JEAN-PIERRE KAHANE}

Let $E$ be a convex set in $R^{p}, d \mu$ a positive bounded measure on $E$, $\Phi(r)$ a decreasing function of $r>0$, which is twice continuously differentiable and summable near 0 , and $V(M)=\int \Phi\left(r_{M P}\right) d \mu_{P}$ the potential of $d \mu$ with respect to $\Phi$ at the point $M\left(M \in R^{p}\right)$. Our purpose is to describe some properties of the "equipotential surfaces" (which are "curves" for $p=2$ )

$$
\left(S_{\lambda}\right)=\{M \text { such that } V(M)=\lambda\}
$$

in relation with $E$, and to generalize some results of J. L. Walsh [1] which concern the case $p=2, \Phi(r)=\log 1 / r$.

Theorem 1. Given $M \notin E$ and $\lambda=V(M)$, let $(N)$ be the normal to $\left(S_{\lambda}\right)$ at the point $M$. Then $(N)$ intersects $E$.

Theorem 2. Suppose $\Phi(r)$ is convex, and let $N$ be the point of $E \cap(N)$ nearest to $M$. Then, in the neighbourhood of $M,\left(S_{\lambda}\right)$ does not intersect the open ball of centre $N$ and radius $N M$.

THEOREM 3. Suppose moreover $\Phi^{\prime \prime}(r) / \Phi^{\prime}(r) \geqq-(\alpha+1) / r(\alpha \geqq 0)$; for example, $\Phi(r)=\log 1 / r$ if $\alpha=0$, or $\Phi(r)=r^{-\alpha}$ if $\alpha>0$. Then, in the neighbourhood of $M,\left(S_{\lambda}\right)$ does not intersect any open ball of radius $\leqq N M /(\alpha+1)$ tangent to $\left(S_{\lambda}\right)$ at $M$. Moreover, if $E$ is compact and if the distance $d$ between $\left(S_{\lambda}\right)$ and $E$ is larger than $(\alpha+2)^{-1 / 2} \Delta, \Delta$ being the diameter of $E$, then $\left(S_{\lambda}\right)$ is a convex surface.

The proofs are quite elementary. Let us write $\Phi(r)=\phi\left(r^{2}\right)$. Then $d V(M)=2 \boldsymbol{N} \cdot \boldsymbol{d} M$ with

$$
\boldsymbol{N}=\int \boldsymbol{P M} \phi^{\prime}\left(\boldsymbol{P} \boldsymbol{M}^{2}\right) d \mu_{P}
$$

Now $(N)$ contains $Q$ defined by

$$
N=Q M \int \phi^{\prime}\left(P M^{2}\right) d \mu_{P}
$$

As $\phi^{\prime} \leqq 0$ and $E$ is convex, we have $Q \in E$, which proves Theorem 1 .

Let us consider now a curve $(C)$ on $\left(S_{\lambda}\right)$ through $M$, such that the center of curvature $C$ at $M$ lies on $(N)$. The conclusion of Theorem 2

Received by the editors May 25, 1961. 
expresses that $C$ is not between $N$ and $M$. Let $s$ be the arc length and $M(s)$ the current points on $(C), t=d M(s) / d s, n / R=d t / d s$ with $|n|=1$; for $s=s_{0}$, we get $M\left(s_{0}\right)=M$ and $M C=n R$; all calculations below are made at $s_{0}$. From $t \cdot N \equiv 0$ there results

$$
\frac{n}{R} \cdot N+t \cdot \frac{d N}{d s}=0
$$

with

$$
\frac{d \boldsymbol{N}}{d s}=t \int \phi^{\prime}\left(P M^{2}\right) d \mu_{P}+2 \int P M \phi^{\prime \prime}\left(P M^{2}\right)(P M \cdot t) d \mu_{P} .
$$

Therefore

$$
\frac{(M Q)^{-}}{(M C)^{-}}=\frac{n \cdot M Q}{R}=1+2 \frac{\int(P M \cdot t)^{2} \phi^{\prime \prime}\left(P M^{2}\right) d \mu_{P}}{\int \phi^{\prime}\left(P M^{2}\right) d \mu_{P}} .
$$

If $\Phi$ is convex, so is $\phi$; therefore

$$
\frac{(M Q)^{-}}{(M C)^{-}} \leqq 1
$$

and $C$ does not belong to the interval $M Q$, which proves Theorem 2 .

The assumption $\Phi^{\prime \prime}(r) / \Phi^{\prime}(r) \geqq-(\alpha+1) / r$ can be written as $\phi^{\prime \prime}\left(r^{2}\right) / \phi^{\prime}\left(r^{2}\right) \geqq-(\alpha+2) / 2 r^{2}$. Majorising $(P M \cdot t)^{2}$ by $P M^{2}$ and $\Delta^{2}$ respectively, we get from (1)

$$
\frac{(M Q)^{-}}{(M C)^{-}} \geqq-1-\alpha
$$

and

$$
\frac{(M Q)^{-}}{(M C)^{-}} \geqq 1-\frac{\alpha+2}{d^{2}} \Delta^{2} .
$$

(3), together with (2), proves the first part of Theorem 3, and (4) the second one.

\section{BibLIOGRAPHY}

1. J. L. Walsh, Amer. Math. Monthly 42 (1935), 1-17.

Faculte des Sciences, Paris, France 